\title{
Configural representations in spatial working memory: modulation by perceptual segregation and voluntary attention
}

\author{
Leon Gmeindl • James K. Nelson • Timothy Wiggin • \\ Patricia A. Reuter-Lorenz
}

Published online: 15 July 2011

(C) Psychonomic Society, Inc. 2011

\begin{abstract}
In what form are multiple spatial locations represented in working memory? The present study revealed that people often maintain the configural properties (interitem relationships) of visuospatial stimuli even when this information is explicitly task-irrelevant. However, the results also indicated that the voluntary allocation of selective attention prior to stimulus presentation, as well as feature-based perceptual segregation of relevant from irrelevant stimuli, can eliminate the influence of stimulus configuration on location-change detection performance. In contrast, voluntary attention cued to the relevant target location following presentation of the stimulus array failed to attenuate these influences. Thus, whereas voluntary selective attention can isolate or prevent the encoding of irrelevant stimulus locations and configural properties, people, perhaps due to limitations in attentional resources, reliably fail to isolate or suppress configural representations that have been encoded into working memory.
\end{abstract}

Keywords Visual working memory · Short-term memory Attention · Grouping

Electronic supplementary material The online version of this article (doi:10.3758/s13414-011-0180-0) contains supplementary material, which is available to authorized users.

L. Gmeindl · J. K. Nelson · T. Wiggin · P. A. Reuter-Lorenz

Department of Psychology, University of Michigan,

Ann Arbor, MI, USA

L. Gmeindl ( $\square)$

Psychological and Brain Sciences, Johns Hopkins University,

Ames Hall, 3400 N. Charles Street,

Baltimore, MD 21218-2680, USA

e-mail: gmeindl@jhu.edu
How are spatial locations coded in working memory (WM)? Studies of behavior and brain activity indicate that attention directed selectively to the locations of previously presented target stimuli plays a critical role in the maintenance of spatial information in WM. For example, discrimination of a stimulus is speeded when it is presented at the location of a rehearsed spatial location during a delay period (Awh, Jonides, \& Reuter-Lorenz, 1998), and a decline in recall and recognition performance is observed when attention is drawn away from target locations (Awh et al., 1998; Smyth, 1996; Smyth \& Scholey, 1994; Van der Stigchel, Merten, Meeter, \& Theeuwes, 2007). Activity is boosted in extrastriate cortex contralateral to the visual hemifield of rehearsed locations (Awh et al., 1999; Postle, Awh, Jonides, Smith, \& D'Esposito, 2004; see also Munneke, Heslenfeld, \& Theeuwes, 2010), and electrophysiological recording has revealed increased amplitudes of sensory-evoked P1 and N1 event-related potentials in response to irrelevant stimuli presented in rehearsed locations (Awh, Anllo-Vento, \& Hillyard, 2000; Jha, 2002).

Furthermore, some studies suggest robust influences of perceptual grouping and spatial configuration ${ }^{1}$ on subjects' detection of changes in items' visuospatial features following a brief delay period. For example, Woodman, Vecera, and Luck (2003) found that the precueing of one colored square led to improved color-change detection for squares that were spatially grouped with the cued square by the Gestalt principles of proximity and connectedness. Jiang, Olson, and Chun (2000), using a memory delay of $907 \mathrm{~ms}$, found that accuracy in detecting a change in an

\footnotetext{
${ }^{1}$ While previous research findings have been compatible with stimulus-driven accounts of configural coding, in our experiments on spatial WM using simple dot stimuli, subjects often reported "mentally connecting the dots," suggesting strategic as well as stimulus-driven sources of configural coding in WM.
} 
item's location was reduced when the locations of some or all of the surrounding items were also changed. Jiang et al. (2000) furthermore found that subjects were better at detecting a color change within a stimulus array when the configuration of items was preserved rather than distorted, even when there were global changes in stimulus locations (i.e., when all elements of the spatial configuration were displaced equally to greater eccentricities).

Because these results indicate that the representation of a given stimulus location is influenced by the relative locations of surrounding items, the question arises of whether configural representation in visuospatial WM is obligatory. That is, can the influence of configural information on the processing of individual spatial locations be overcome, and, if so, under which conditions and by what mechanisms? Jiang et al. (2000) tested whether segregating target items from distractors by color would eliminate the influence of distractor locations on color-change detection. They found that under these conditions the locations of distractors did not reliably influence accuracy for detecting a color change among the targets. Although Jiang et al. (2000) did not independently manipulate stimulus configuration and stimulus displacement in that experiment, their results suggest that feature-based perceptual segregation can override the influence of stimulus configuration on the detection of changes in color (although not necessarily in location). They (see also Jiang, Chun, \& Olson, 2004) also concluded that, in contrast, subjects could not ignore distractors that were not physically distinct from targets (e.g., those not segregated by color), and thus the overall configuration of targets and distractors hindered detection of change in the color of targets.

The studies cited above provide a rich set of findings regarding the representation of spatial information in WM.
The goal of the present study was to further examine the influences of stimulus configuration on WM for stimulus location, and to test the hypotheses that perceptual segregation and voluntary attention can modulate these influences. Here, we report the results of three experiments in which stimulus configurations and stimulus displacements were each manipulated to test whether and under what conditions locations are represented in spatial WM as multilocation configurations.

\section{Experiment 1}

In each trial of Experiment 1, three target dots were presented simultaneously, and following a delay period, memory for the locations of these dots was probed by three circles (Fig. 1). Subjects were instructed to indicate whether all of the probe locations matched all of the target locations. For nonmatch trials, either two or three of the probe locations did not match the target locations; orthogonal to this manipulation of stimulus displacement, the configuration (i.e., the interitem relationships) of target stimuli was either preserved or distorted at the probe phase. This method equated the absolute change in location between the target and probe displays as well as stimulus eccentricity across the two configuration conditions. We hypothesized that location change detection is influenced by both the nature of the stimulus configuration and the degree of spatial overlap between the target array and the test array. We therefore had three predictions regarding the effects of these manipulations: (1) because it should be more difficult for subjects to detect changes in stimulus locations between the target and test arrays when two rather than three stimuli are displaced, the displacement

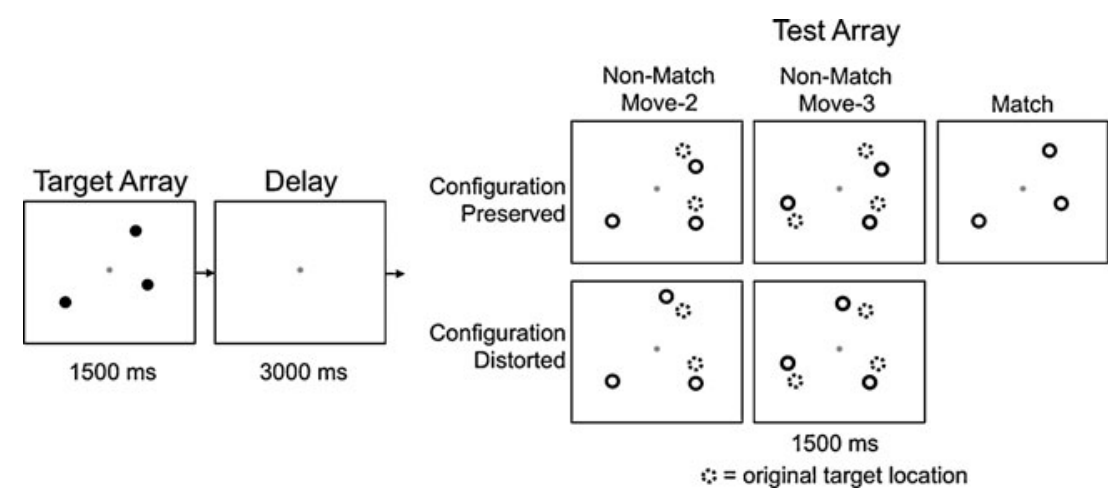

Fig. 1 The trial structure and types of test array presented in Experiment 1. A warning tone and fixation dot were onset $500 \mathrm{~ms}$ prior to the target array. Dotted circles in the test array illustrate the original target locations and were not presented in the experiment. For match test arrays, the locations of probe stimuli presented in the test array matched all three target locations. For nonmatch test arrays,

either two or three probe stimuli were presented in nonmatching locations, and independently, the configuration of the probe stimuli either preserved or distorted the configuration of the target stimuli. For this and subsequent schematic figures (Figs. 3 and 5), stimuli are not drawn to scale 
of two stimuli should result in a higher false-alarm rate (i.e., an increased frequency of incorrect "match" responses) than should the displacement of three stimuli; (2) if subjects' ability to discriminate changes in stimulus location is influenced by the presence or absence of changes in stimulus configuration, the preservation of stimulus configuration should result in an increased false-alarm rate relative to the distortion of stimulus configuration; and (3) these factors should interact such that the increase in false-alarm rate due to displacing two rather than three stimuli should be larger for preserved than for distorted stimulus configurations.

\section{Method}

Subjects A group of 10 subjects (6 female, 4 male; 18 to 22 years of age, $M=19.0$ years) were paid a minimum of $\$ 15.00$ for participation. Subjects received a $\$ 0.02$ bonus for each correct response and a $\$ 0.02$ penalty for each incorrect response. Subjects were videotaped during the performance of the task and were told that it was extremely important to maintain fixation during trials. The videotapes were inspected visually to verify compliance with these instructions; 1 additional subject was omitted from further analysis due to noncompliance.

Apparatus and stimuli A 17-in. CRT monitor with resolution set to $640 \times 480$ pixels was used, and stimulus presentation and data collection were controlled by E-Prime software (Psychology Software Tools, Pittsburgh, PA). A chinrest ensured a constant viewing distance of $\sim 43 \mathrm{~cm}$. Responses were made by pressing the " $\mathrm{z}$ " and " " keyboard keys with the left and right index fingers, respectively.

A small dot (8-point solid dot, Wingdings font) presented at the center of the computer screen served as a fixation point. The procedure for generating target and test arrays is reported in the supplementary materials (see Figs. S1 and S2), but a general description follows. Each target set consisted of three dots appearing at unpredictable locations. Five types of test array were generated for each of these target sets: match, move-2/ preserved configuration, move-2/distorted configuration, move-3/preserved configuration, and move-3/distorted configuration (Fig. 1). In the match condition, all three probe locations matched the three target locations, and therefore the correct response was to indicate a match; in the remaining conditions, either two or three of the probe locations did not match the locations of the corresponding targets, and therefore the correct response was to indicate a nonmatch.

In the move-3/preserved configuration condition, all probe items were displaced from the corresponding target locations, but the target configuration (interitem relation- ships) was preserved via rotation of the stimulus array (Figs. S1a-S1c). In the move-3/distorted configuration condition, probe items were similarly displaced from the corresponding target locations via rotation, but one probe item was displaced in a different direction (Figs. S1d, S1e), resulting in a distortion of the target configuration. The eccentricities (relative to the fixation point) of probe items and the magnitude of probe displacements were equated between the move-3/preserved configuration and move-3/ distorted configuration conditions.

In the move-2/preserved configuration condition, one of the target locations was chosen randomly as the point of rotation about which the other two probes were displaced from their corresponding target locations, thereby preserving the target configuration (Figs. S2a-S2c). In the move-2/distorted configuration condition, in contrast, one of the probes was displaced in a different direction, resulting in a distortion of the target configuration (Figs. S2d, S2e). As in the move-3 conditions, stimulus eccentricity and the magnitude of probe displacements were equated between the move- $2 /$ preserved configuration and move-2/distorted configuration conditions.

Procedure A red fixation point and a warning tone were presented simultaneously at the beginning of each trial; the fixation point remained red until the end of the trial. A total of $500 \mathrm{~ms}$ later, three black target dots (12-point solid dots, Wingdings font) were presented simultaneously and remained on the screen for $1,500 \mathrm{~ms}$ (Fig. 1). After a 3,000-ms retention interval, during which only the fixation point was presented, three black probe circles (14-point unfilled circles, Wingdings font) appeared for 1,500 ms. The fixation point was presented in green during the subsequent $1,500-\mathrm{ms}$ intertrial interval (ITI) to allow subjects to blink or to move their eyes. Following the onset of the test array, subjects indicated by pressing one of two response keys whether all three probe stimuli occurred in the same locations as the three target stimuli. The instructions to subjects emphasized accuracy over speed of responses, and responses were recorded throughout the 3 -s period from the onset of the test array until the end of the ITI, to ensure that even long-latency responses were recorded. Subjects were informed that match and nonmatch trials were equally probable. Match test arrays appeared in $50 \%$ of trials, and each of the four types of nonmatch test arrays appeared approximately equally often $(12 \%-13 \%)$ in the remaining $50 \%$ of trials.

After completing one block of 8 practice trials, subjects completed six blocks of 64 trials each (32 trials with match test arrays and 8 trials with each type of the nonmatch test arrays, randomly interleaved), for a total of 384 test trials. 
Results

ANOVAs specifying within-subjects factors of Stimulus Configuration (preserved, distorted) and Stimulus Displacement (move-2, move-3) were performed separately for the error rate and RT data from nonmatch conditions; data from the match condition were not entered into the ANOVAs because this condition did not vary across the levels of either factor.

The false-alarm rate (i.e., the proportion of nonmatch trials in which subjects indicated a match) was higher when stimulus configurations were preserved than when they were distorted $[.33$ vs. .21 , respectively; $F(1,9)=37.276$, $p<.001]$. The false-alarm rate was also higher when only two stimuli were displaced than when three stimuli were displaced $[.32$ vs. .22 , respectively; $F(1,9)=$ 19.283, $p=.002]$. Moreover, these factors interacted such that the increase in the false-alarm rate from move- 3 to move- 2 conditions was larger when stimulus configurations were preserved rather than distorted $[F(1,9)=21.981, p=$ .001; see Fig. 2].

A similar pattern of results emerged for correct nonmatch RT data, even though the instructions emphasized accuracy over the speed of responses. Test arrays that preserved the stimulus configuration took longer to reject than test arrays that distorted the stimulus configuration [916 vs. $864 \mathrm{~ms}$, respectively; $F(1,9)=8.814, p=.021]$. Move-2 test arrays took longer to reject than move-3 test arrays [917 vs. $864 \mathrm{~ms}$, respectively; $F(1,9)=9.548, p=.018]$. There was a borderline significant interaction of these factors $[F(1,9)=$ $5.426, p=.053]$, in a manner consistent with the pattern of error rates. Of particular importance, there was no evidence for speed-accuracy trade-offs in Experiment 1: Conditions producing longer mean RTs also produced increased false-alarm rates.

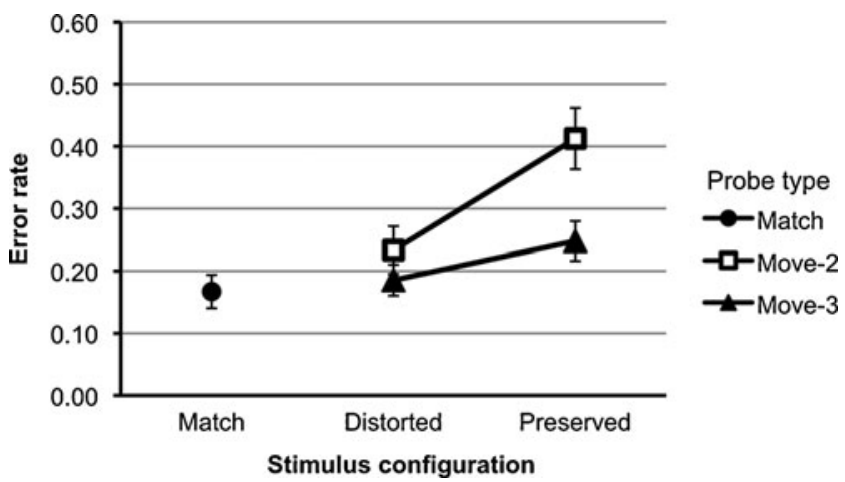

Fig. 2 Experiment 1: Mean $( \pm S E)$ error rates (i.e., proportions of misses for match trials, proportions of false alarms for move- 2 and move- 3 nonmatch trials) as a function of stimulus configuration and probe type
Discussion

The three predictions of Experiment 1 were confirmed: (1) The displacement of two stimuli resulted in a higher false-alarm rate than did the displacement of three stimuli. (2) The preservation of stimulus configuration resulted in a higher false-alarm rate than did the distortion of stimulus configuration. (3) These factors interacted such that the increase in false-alarm rate due to displacing two rather than three stimuli was larger for preserved than for distorted stimulus configurations.

These findings extend those of Jiang et al. (2000), who provided evidence that concomitant changes in the configuration and locations of items impaired locationchange detection. Based on the results of our independent manipulation of these two factors, we concluded that stimulus configuration and stimulus displacement jointly influence location-change detection performance. We speculate that these factors influence processing at a decision stage at which the evidence for spatial mismatch is weighed - where a strong match signal due to preserved stimulus configuration can override a weak mismatch signal due to a partial spatial overlap between target and test arrays (e.g., in the move-2/preserved configuration condition).

\section{Experiment 2}

We began next to study the roles of attention and perceptual grouping in the representation of spatial locations in WM. We considered two reasons why stimulus configuration might have such a strong influence over location-change detection, as evidenced by the results of Experiment 1 and those of Jiang et al. (2000): First, subjects might strategically "chunk" stimulus locations by relying more heavily on a configural form of representation than on representations of individual spatial locations (Bor, Duncan, \& Owen, 2001; Bor, Duncan, Wiseman, \& Owen, 2003). Configural coding also might be automatic (i.e., not requiring effortful, controlled processing). Configural coding of multiple items, be it strategic or automatic, might occur because chunking can reduce memory load. If configural representation of spatial information is in fact the result of strategic processing, then subjects might instead maintain individual locations as configuration-independent representations when required to evaluate the match/nonmatch status of only a single probe item rather than of the entire test array. Relevant to this possibility, Woodman et al. (2003), in their study on the influences of perceptual grouping on WM, suggested that items that are grouped together are stored together, but Woodman et al. also speculated that these influences might be modulated by 
task demands. Accordingly, Experiment 2 required the evaluation of only a single relevant probe item; the match/ nonmatch status of the two other probe items and the preserved/distorted status of the overall stimulus configuration were uninformative and task-irrelevant. Furthermore, subjects were explicitly told that they could ignore the irrelevant probe distractors.

The second possibility we considered is that a match in nonspatial visual features (viz. color) across target and probe arrays, and among items within the probe array, might bias subjects toward the use of configural information when deciding whether a change in location has occurred. For example, an attentional template (Duncan \& Humphreys, 1989) for processing black dots at the target phase may result in the processing of (e.g., spread of attention to) all black stimuli at the probe phase, even in cases where the correct change-detection decision could be achieved based on a single probe item. Therefore, in one condition of Experiment 2, the relevant target and the relevant probe were each presented as a color singleton, thus perceptually segregating the relevant target and probe items from distractors that subjects were allowed to ignore.

In addition, the perceptual segregation of relevant from irrelevant items by color was manipulated independently for target and probe phases across different conditions of Experiment 2, to examine the influences of configural processing at each of these phases. Without perceptual segregation of the relevant target from distractors (i.e., when no target singleton is presented), it is possible that the encoding and maintenance of configural information present in the target array facilitates configural processing of the test array. This may attenuate any benefit of perceptual segregation provided by the color singleton at the probe phase. In contrast, if the relevant target item is in fact presented as a color singleton, then configural coding of the target array in WM might be minimized. In that case, the influence of stimulus configuration on performance might be eliminated, regardless of whether the relevant probe item is perceptually segregated from distractors. Experiment 2 therefore systematically varied the presence of color singletons at the target and probe phases in order to assess these possibilities.

The fact that memory load is reduced when the target is presented as a color singleton (because the singleton location is the only one that subjects are instructed to remember) has two noteworthy implications: First, the target singleton should allow subjects to rehearse only the task-relevant location, and, second, the overall level of performance should improve, because only one location held in WM would need to be consulted at the probe phase. As a result, the persistence of an influence of stimulus configuration on performance even in this relatively easy condition would highlight the strength of the configural influence on location-change detection performance, and would perhaps suggest that the configural representation of spatial locations in WM is automatic, even when targets are perceptually segregated from distractors.

In one condition, which for simplicity we refer to as the red-red condition, a task-relevant red target color singleton was presented among black distractors at the target phase, and a task-relevant, red probe color singleton was presented among black distractors at the probe phase (see Fig. 3). In the black-red condition, three black targets were followed by a red probe color singleton among black distractors. Finally, in the red-black condition, a red target color singleton was presented among black distractors at the target phase, and all probe items were black. The latter condition allowed us to test whether circumstances that permit one to restrict attention to a single target item would be sufficient to eliminate the influence of stimulus configuration on performance, despite the absence of a color-singleton probe; that outcome would suggest that the representation of configural information in WM had been suppressed or isolated relatively early in the trial, perhaps during the encoding of the target's location.

\section{Method}

Subjects The data from 16 subjects ( 7 female, 9 male; 18 24 years of age, $M=21.0$ years) were included in the analyses presented below. An additional 15 subjects were
Red-Red

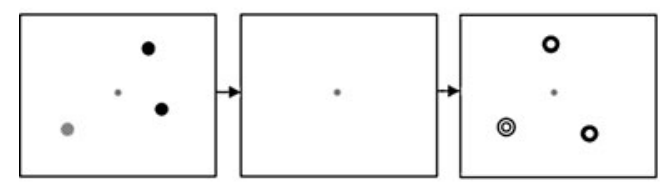

Black-Red

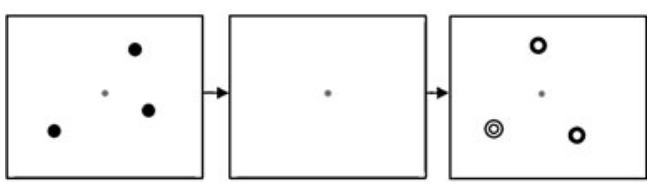

Red-Black

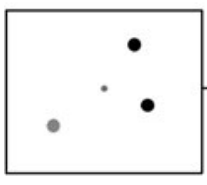

Target Array
$1500 \mathrm{~ms}$



Delay

$3000 \mathrm{~ms}$

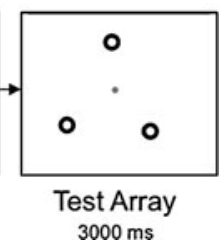

Fig. 3 Simplified trial structure and conditions presented in Experiment 2. In separate blocked conditions, a task-relevant red color singleton was presented among black distractors in the target and/or test arrays. Color-singleton targets are represented here by gray stippled dots, and color-singleton probes are represented here by concentric circles 
excluded due to eye movements in one or more task conditions (see the next section for additional details). Payment was determined as in Experiment 1.

Apparatus, stimuli, and procedure The apparatus and stimuli were identical to those in Experiment 1, except that target and/or probe color singletons were red and all other items were black (see Fig. 3): In the black-red condition, the relevant probe item was red and all other items were black; subjects were instructed to indicate whether the probe color singleton occurred at the location of a target. In the red-black condition, the relevant target item was red and all other items were black; subjects were instructed to indicate whether a probe item occurred at the location of the target color singleton. In the red-red condition, a relevant red color singleton was presented among black distractors at both target and probe phases; subjects were instructed to indicate whether the probe color singleton occurred at the location of the target color singleton. These three color-singleton conditions were presented in a blocked fashion, as described below. All test arrays were generated as in the move-2/ preserved configuration and move-2/distorted configuration conditions of Experiment 1. However, in Experiment 2 , match trials were those in which the relevant probe item matched the location of the corresponding target item; nonmatch trials were those in which the location of the relevant probe item was displaced from the location of the corresponding target item. The location match/nonmatch status of the two probe distractors and the preserved/ distorted status of the overall stimulus configuration were uninformative and task-irrelevant. Subjects were explicitly told that they could ignore the irrelevant probe distractors.

After completing eight practice trials, subjects completed 12 blocks of trials. Each color-singleton condition (blackred, red-black, red-red) was presented in four successive blocks of 32 trials each [8 trials with each type of test array (match/preserved configuration, nonmatch/preserved configuration, match/distorted configuration, nonmatch/distorted configuration) randomly interleaved], for a total of 384 test trials. The order of color-singleton conditions was randomized across subjects. Other details of the procedure were identical to those of Experiment 1.

Because color singletons might be effective at eliciting reflexive saccades, and because one strategy for ignoring distractor items could be to voluntarily shift gaze to the color singleton, additional measures were taken in this and the subsequent experiment to minimize the possibility that any effects of color singletons (or of cues in Exp. 3) on performance were not due to eye movements away from the fixation stimulus. As in the previous experiment, subjects were videotaped during performance of the task, and the importance of maintaining fixation throughout each trial was emphasized. Each subject's videotape was visually inspected by a trained research assistant, who scored every trial according to whether any detectable deviation from the fixation stimulus occurred from the onset of the target array (Exp. 2) or from the pretarget cue (Exp. 3) through presentation of the test array. Subjects making eye movements on more than $15 \%$ of trials during any of the three color-singleton (Exp. 2) or cueing (Exp. 3) conditions were excluded from the analyses reported below. Nevertheless, performance did not differ reliably between included and excluded subjects, indicating that subjects either did not strategically employ, or did not benefit from, shifts in gaze. ${ }^{2}$

\section{Results}

Mean error rates are plotted in Fig. 4. In the black-red condition, more errors were made when stimulus configurations were preserved than when they were distorted $[F(1,15)=24.523, p<.001]$, more errors were made on nonmatch than on match trials $[F(1,15)=42.196, p<.001]$, and there was a reliable interaction between stimulus configuration and probe type $[F(1,15)=12.305, p=$ .003], such that the increase in error rates from match to nonmatch trials was greater for preserved than for distorted configurations. An analysis of simple main effects revealed that the effect of stimulus configuration was reliable for nonmatch trials $[F(1,15)=27.318, p<.001]$ but not for match trials $[F(1,15)=0.043, p=.839]$.

A similar pattern of results was observed for the redblack condition: More errors were made when stimulus configurations were preserved than when they were distorted $[F(1,15)=25.246, p<.001]$, more errors were made on nonmatch than on match trials $[F(1,15)=20.695$, $p<.001]$, and there was a reliable interaction between stimulus configuration and probe type $[F(1,15)=5.088$, $p=.039]$, such that the increase in error rates from match to nonmatch trials was greater for preserved than for distorted configurations. An analysis of simple main effects revealed that the effect of stimulus configuration was reliable for nonmatch trials $[F(1,15)=25.697, p<.001]$ but not for match trials $[F(1,15)=2.137, p=.164]$.

In contrast, in the red-red condition, whereas more errors were made on nonmatch than on match trials $[F(1,15)=$ $9.868, p=.007]$, there was no reliable difference between

\footnotetext{
${ }^{2}$ Given the relatively large number of excluded subjects resulting from our eye-movement exclusion criteria, we tested whether results from included subjects were atypical. We analyzed the data from all subjects excluded due to eye movements, and then added Group (included vs. excluded subjects) as a between-subjects factor to all ANOVAs reported here. All statistically significant effects reported in the main text remained significant, and no nonsignificant effects became significant. Furthermore, Group did not interact with any factor analyzed.
} 
Fig. 4 Experiment 2: Mean $( \pm S E)$ error rates as a function of singleton condition, stimulus configuration, and probe type



error rates when stimulus configurations were preserved rather than distorted $[F(1,15)=0.600, p=.451]$, nor was there a reliable interaction between stimulus configuration and probe type $[F(1,15)=0.597, p=.452]$.

The difference in the patterns of error rates among the three color-singleton conditions was confirmed by a reliable three-way interaction $[F(2,30)=8.407, p=.001] .^{3}$ Also, mean error rates varied by color-singleton condition $[F(2$, $30)=19.726, p<.001]$; pairwise comparisons indicated that error rates were higher for black-red than for both redblack $[F(1,15)=25.080, p<.001]$ and red-red $[F(1,15)=$ $31.293, p<.001]$ conditions.

Mean RTs for correct responses were longer for nonmatch trials (931 ms) than for match trials [ $898 \mathrm{~ms} ; F(1,15)=7.961$, $p=.013]$. Mean RTs also varied by color-singleton condition [black-red, 1,016 ms; red-black, $873 \mathrm{~ms}$; red-red, $854 \mathrm{~ms} ; F$ $(2,30)=17.301, p<.001]$. Of particular importance, however, there were no reliable interactions between stimulus configuration and probe type for any of the colorsingleton conditions. Furthermore, there was no evidence of speed-accuracy trade-offs in Experiment 2.

\section{Discussion}

Several findings from Experiment 2 warrant discussion. First, in the black-red condition, whereas the preservation versus distortion of the stimulus configuration had little or no effect when the relevant probe appeared in the same location as its corresponding target (match trials), preservation of the configuration did lead subjects to make more false-alarm errors during nonmatch trials. These results might reflect a two-stage decision process whereby the evidence for spatial mismatch between the relevant target and probe locations is first weighed, and then if there is only weak evidence for mismatch, the relative positions of nominally task-irrelevant items are evaluated and contribute to the subject's decision. The fact that segregating the relevant probe item from

\footnotetext{
$\overline{{ }^{3} \text { An analysis of }} d^{\prime}$ (Tanner \& Swets, 1954) indicated a similar pattern of results: Mean $d^{\prime}$ was reliably lower for preserved than for distorted stimulus configurations for black-red $(p=.001)$ and red-black $(p=$ $.003)$ conditions, but not for the red-red condition $(p=.330)$.
}

distractors failed to eliminate the influence of stimulus configuration in this condition is discussed further below.

Second, performance was significantly improved in both conditions (red-black and red-red) in which the memory load was reduced to a single location by the presentation of a colorsingleton target. Nevertheless, this manipulation was insufficient to eliminate the influence of stimulus configuration on performance in the absence of a color-singleton probe (redblack). This result highlights the tenacity of the configural influence on location-change detection performance. However, the influence of configuration was in fact attenuated in redblack relative to black-red, a result that is consistent with the hypothesis that reducing spatial memory load reduces subjects' reliance on configural information. People may employ the configural chunking or reorganization of locations when faced with relatively heavy spatial memory loads (Gmeindl, Walsh, \& Courtney, 2011). Alternatively, or in addition, the presentation of the target as a color singleton likely attenuates the configural coding of the target array due to preattentive influences of perceptual segregation, and any bottom-up or contingent capture of attention by the salient target colorsingleton (for a review, see Egeth \& Yantis, 1997) may also help to attenuate configural coding of the target array.

In contrast to the results from the black-red and red-black conditions, perceptual segregation of relevant from irrelevant items at both target and probe phases (red-red) virtually eliminated the influence of stimulus configuration, suggesting that the representation of configural information encountered during target and probe phases was either suppressed or isolated from configuration-independent representations in WM.

The latter conclusion is further supported by another finding from Experiment 2 that at first seems counterintuitive. One might expect that when only a single target location is indicated as task-relevant upon presentation of the target array (e.g., in red-black and red-red conditions), filtering out stimuli at other locations should optimize discrimination of change in the relevant stimulus location. Yet, we found that more false alarms were made in the context of distorted configurations when a relevant color singleton was also presented at the probe phase (red-red) as compared to when no color singleton was presented at the probe phase (red-black), $t(15)=2.58, p=.021$. However, 
this paradox is easily resolved. Specifically, it appears from Experiment 2 that when configural information is evaluated, distortion of the configuration leads congruently to correct rejections when there is a mismatch in location between the task-relevant target and probe items, coinciding with a decreased false-alarm rate (as for the red-black, distorted configuration condition). If, however, one eliminates the evaluation of configural information, correct rejections are not facilitated by distorted configurations, resulting in a relatively higher false-alarm rate (as for the red-red, distorted configuration condition).

\section{Experiment 3}

The results of Experiment 2 indicate that perceptual segregation of relevant from irrelevant stimuli at both target and probe phases is sufficient for eliminating the influences of spatial configuration on location-change detection. In Experiment 3, we investigated whether voluntary attention can also attenuate the influences of spatial configuration on location-change detection. Specifically, we hypothesized that voluntary selective attention engaged during the encoding of relevant spatial information into WM can suppress or isolate the representation of configural information. Therefore, in one condition, rather than segregating the relevant target stimulus from distractors by color, we instead presented prior to target presentation a cue at fixation that indicated the relevant visual quadrant; subjects were required to remember only the location of the stimulus subsequently appearing within that quadrant. If the voluntary allocation of selective attention is as effective at isolating or suppressing the encoding of stimulus configuration as is the perceptual segregation afforded by a color-singleton target, then the influence of stimulus configuration on locationchange detection should be absent in this condition, as it was in the red-red condition of Experiment 2. However, might attention also isolate or suppress the configural representation of spatial information after a stimulus array has been encoded? Perhaps attention can be voluntarily restricted to one of several locations held in WM, at the expense of configural representation. If so, presentation of a posttarget cue should likewise result in an attenuated influence of stimulus configuration on location-change detection performance. To test these hypotheses, in Experiment 3 we presented a central arrow cue either before or after the target phase, in a blocked fashion; in a third, baseline condition, the arrow cues were replaced with uninformative, neutral cues.

Method

Subjects A group of 28 subjects (13 female, 15 male; 18 27 years of age, $M=21.0$ years) were included in the analyses reported below. An additional 8 subjects were excluded for excessive eye movements. Payment was determined as in Experiment 1.

Apparatus, stimuli, and procedure The same apparatus and stimuli were used in Experiment 3 as in the black-red condition of Experiment 2, except for the following changes. Two types of central cue were presented in Experiment 3 (see Fig. 5). The uninformative, neutral cue was a red "+" symbol 17 pixels in height and width, presented at the center of the computer screen. The informative cue was a similarly sized red arrow (i.e., comprising line segments the same length as those constituting the neutral cue) pointing in one of four directions (upper left, upper right, lower left, lower right), presented at the center of the screen. The informative cue indicated the relevant visual quadrant; the only target location that subjects were required to remember, and the only probe stimulus subjects were required to evaluate, was presented within the cued quadrant. Subjects were instructed to indicate whether the location of the probe color singleton (which always occurred within the cued quadrant when an informative cue was provided) matched the location of the corresponding target (which also always occurred within the cued quadrant when an informative cue was provided). Cueing periods immediately preceded and followed the target array. For the cueing period immediately preceding the target array, the cue was presented for $1,000 \mathrm{~ms}$ and was followed by presentation of the fixation point for $500 \mathrm{~ms}$ prior to the onset of the target array. For the cueing period immediately following the target array, the fixation point was presented for $500 \mathrm{~ms}$ and was followed by presentation of the cue for $1,000 \mathrm{~ms}$. A 3,000-ms delay, during which only the fixation point was presented, preceded the onset of the test array.

In the neutral cueing condition, uninformative, neutral cues were presented before and after presentation of the target array. In the pretarget cueing condition, an informative arrow cue was presented before and an uninformative cue was presented after the target array. In the posttarget cueing condition, an uninformative cue was presented before and an informative arrow cue was presented after the target array.

After completing 8 practice trials, subjects completed twelve trial blocks. Each cueing condition was presented in four successive blocks of 32 trials each [8 trials with each type of test array (match/preserved configuration, nonmatch/preserved configuration, match/ distorted configuration, nonmatch/distorted configuration), randomly interleaved], for a total of 384 test trials. The order of cueing conditions was randomized across subjects. 
Fig. 5 Simplified trial structure and conditions presented in Experiment 3. In separate blocked conditions, a central arrow cue indicating the relevant quadrant was presented either before or after the target array, or otherwise was replaced with an uninformative neutral cue. A task-relevant red colorsingleton probe was presented among black distractors in the test display
Neutral Cueing

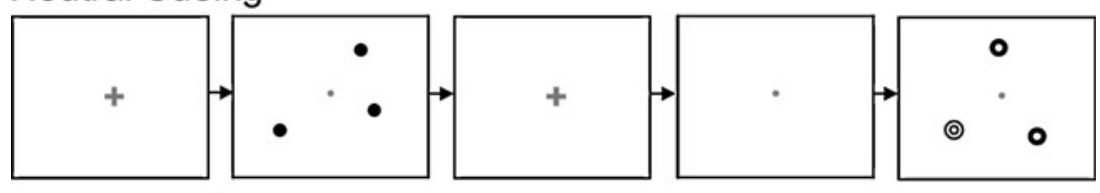

Pre-Target Cueing

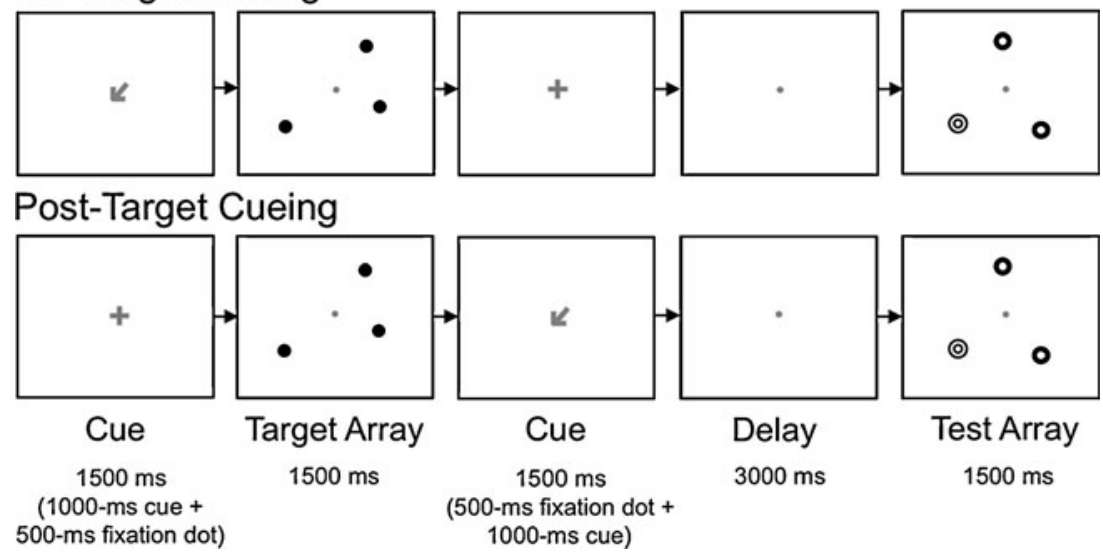

Results

Mean error rates are plotted in Fig. 6. In the neutral cueing condition, more errors were made on nonmatch than on match trials $[F(1,27)=13.761, p=.001]$, and there was a reliable interaction between stimulus configuration and probe type $[F(1,27)=18.733, p<.001]$, such that the increase in error rates from match to nonmatch trials was greater for preserved than for distorted configurations. An analysis of simple main effects revealed that the effect of stimulus configuration was reliable for nonmatch trials $[F(1,27)=27.136, p<.001]$ but not for match trials $[F(1,27)=1.666, p=.208]$.

In the posttarget cueing condition, more errors were made on nonmatch than on match trials $[F(1,27)=19.327$, $p<.001]$, more errors were made when stimulus configurations were preserved rather than distorted $[F(1,27)=$ $6.364, p=.018]$, and there was a reliable interaction between stimulus configuration and probe type $[F(1,27)=$ $8.730, p=.006]$, such that the increase in error rate from match to nonmatch trials was greater for preserved than for distorted configurations. An analysis of simple main effects revealed that the effect of stimulus configuration was reliable for nonmatch trials $[F(1,27)=20.164, p<.001]$ but not for match trials $[F(1,27)=0.680, p=.417]$.

In contrast, in the pretarget cueing condition, whereas more errors were made on nonmatch than on match trials $[F(1,27)=13.964, p=.001]$, there was no reliable difference between error rates when stimulus configurations were preserved rather than distorted $[F(1,27)=$ $0.600, p=.451]$, nor was there a reliable interaction between stimulus configuration and probe type $[F(1,27)<$ $0.001, p=.984]$.
The differences in the patterns of error rates among the three cueing conditions were confirmed by a reliable three-way interaction $[F(2,54)=5.573, p=.006] .{ }^{4}$ Also, mean error rate varied by cueing condition $[F(2,54)=$ 27.980, $p<.001]$, with error rate higher for the neutral cueing condition than for both the pretarget cueing condition $[F(1,27)=16.351, p<.001]$ and the posttarget cueing condition $[F(1,27)=6.297, p=.015]$.

Mean RTs for correct responses did not reliably differ between nonmatch trials $(1,067 \mathrm{~ms})$ and match trials $(1,049 \mathrm{~ms}), F(1,27)=2.571, p=.121$. Mean RTs varied by cueing condition (neutral cueing, 1,149 ms; pretarget cueing, 1,012 ms; posttarget cueing, $1,012 \mathrm{~ms}), F(2,54)=$ 27.504, $p<.001$. Of particular importance, a reliable interaction between stimulus configuration and probe type was observed only for the neutral cueing condition (nonmatch/distorted, 1,132 ms; nonmatch/preserved, 1,163 ms; match/distorted, 1,178 ms; match/preserved, 1,125 ms), $F(1$, $27)=18.733, p<.001$; the pattern of mean RTs in this condition was therefore consistent with the pattern of error rates: Within both match and nonmatch trials, longer mean RTs coincided with higher error rates. There was no evidence for speed-accuracy trade-offs in Experiment 3.

\section{Discussion}

In Experiment 3, the presentation of an informative central cue prior to the target array eliminated the influences of

\footnotetext{
${ }^{4}$ An analysis of $D^{\prime}$ indicated a similar pattern of results: Mean $d^{\prime}$ was reliably lower for preserved than for distorted stimulus configurations for neutral cueing $(p=.021)$ and posttarget cueing $(p=.039)$ conditions, but not for the pretarget cueing condition $(p=.293)$.
} 
Fig. 6 Experiment 3: Mean

$( \pm S E)$ error rates as a function of cueing condition, stimulus configuration, and probe type



stimulus configuration on location-change detection performance, as had perceptual segregation of relevant stimuli from distractors by color in Experiment 2. These results are consistent with the hypothesis that the voluntary allocation of selective attention prior to stimulus presentation isolates or suppresses the encoding of irrelevant stimulus locations and configural information into WM. ${ }^{5}$ In contrast, providing an informative central cue only $2 \mathrm{~s}$ after the onset of the target array increased overall performance (see Lepsien \& Nobre, 2006) but had little if any mitigating effect on the influences of stimulus configuration on location-change detection performance. These findings indicate that whereas voluntary attention can isolate target-location representations from configural representations or prevent the encoding of distractor locations or configural properties into WM, people, perhaps due to limitations in attentional resources, reliably fail to isolate or suppress configural representations that have been encoded into WM.

\section{General discussion}

Our findings indicate that, consistent with results from color-change detection studies employing brief retention intervals (approximately $900 \mathrm{~ms}$; Jiang et al., 2000; Woodman et al., 2003), configural information is maintained in WM over longer intervals (3-4.5 s) that increase the demand for active maintenance; this is the case even when stimulus configuration is explicitly task-irrelevant.

\footnotetext{
${ }^{5}$ To verify that voluntary attention allocated prior to the target array is sufficient to attenuate configural influences in the absence of perceptual segregation, we conducted an additional experiment $(N=$ 22) in which the pretarget cueing condition of Experiment 3 was given, as well as a condition in which the same arrow cue appeared (redundantly) both before the target array and before the test array, and all targets and probes were black in the latter condition. Otherwise, the conditions were identical. The pattern of results from the pretarget cueing condition of Experiment 3 was replicated: For each of these conditions, whereas more errors were made on nonmatch than on match trials $(p s<.001)$, there was no reliable main effect of stimulus configuration (preserved vs. distorted) nor a reliable interaction between stimulus configuration and probe type (match vs. nonmatch; all $p \mathrm{~s}>.05)$, consistent with the above hypothesis.
}

Results from some previous studies suggested that the configural properties of stimulus arrays influence the ability to detect changes in visual features. For example, Jiang et al. (2000) found that detection of change in the color of target items was impaired when the stimulus configuration changed from target to probe phases. Delvenne and Bruyer (2006) concluded that configural coding and object-based forms of representation both facilitate detection of changes in the color and orientation of array elements. However, while those particular experiments addressed the role of configural coding in visual WM, they did not test whether stimulus configuration influences WM for spatial locations. Furthermore, in the one experiment of Jiang et al. (2000, Exp. 2a) that did test whether stimulus configuration influenced location memory, changes in configuration were confounded with changes in locations. In contrast to previous experiments, subjects in our study were required to detect changes in spatial location, and the changes in stimulus configuration were manipulated independently of changes in stimulus locations. Based on the results of our manipulations of stimulus configuration and stimulus displacement, we conclude that these factors jointly influence location-change detection performance. $^{6} \mathrm{We}$ further speculate that these factors influence processing at a decision stage at which the evidence for spatial mismatch

\footnotetext{
${ }^{6}$ The possibility of simple verbal recoding of target locations (e.g., "1 o'clock," "3 o'clock," "7 o'clock") does not seem consistent with the observed influences of both stimulus configuration and stimulus displacement on performance. First, because the displacements of nonmatching test items from their original locations were equivalent (and as small as $1 \mathrm{deg}$ of visual angle) across the two types of configuration, a rather precise representation of the interitem relationships would be required in order to account for the differential effects of preserved versus distorted stimulus configurations. Second, subjects' performance indicated a sensitivity to differences in stimulus displacement as fine as $0.5 \mathrm{deg}$ of visual angle. These findings require a precision of spatial representation beyond that provided by plausible verbal recoding strategies. Finally, the influences of preserved versus distorted stimulus configuration on performance have recently been replicated with a much shorter exposure duration $(250 \mathrm{~ms}$; Boduroglu, Gmeindl, \& Reuter-Lorenz, 2011) that may reduce the likelihood of verbal recoding, and Jiang et al. (2000) found influences of stimulus configuration on color-change detection performance using both short exposure duration $(400 \mathrm{~ms})$ and short retention interval $(907 \mathrm{~ms})$, conditions under which verbal recoding of stimulus locations is highly unlikely.
} 
between stimuli is weighed. A strong match signal due to preserved stimulus configuration might override a weak mismatch signal due to partial spatial overlap between target and test arrays, thereby resulting in false alarms. In contrast, a mismatch signal due to distorted configuration might lead congruently to correct rejections in the presence of relevant stimulus displacements.

In the present study, we also tested the hypothesis that perceptual segregation of relevant from irrelevant stimuli attenuates the influence of overall stimulus configuration on location-change detection performance. We found that segregating a single target item from distractors by color improved performance but did not eliminate the influence of stimulus configuration. However, this influence was in fact eliminated when both relevant target and probe items were perceptually segregated from distractors. These findings suggest that perceptual grouping of relevant with irrelevant items at target and/or probe phases leads people to rely on configural representations in making location-change decisions (see Jiang et al., 2000; Woodman et al., 2003).

Of particular theoretical interest are two novel findings. First, a centrally presented attention cue preceding target presentation was as effective as perceptual segregation by color at eliminating the influences of stimulus configuration on location-change detection performance. This result indicates that the utilization of configural representations is not obligatory; rather, subjects can voluntarily and selectively allocate attention to relevant portions of the visual field, thereby suppressing or isolating the representation of configural information in WM. Second, a central cue presented only $2 \mathrm{~s}$ following target presentation (a delay that is expected to exceed the duration of consolidation in WM; Vogel, Woodman, \& Luck, 2006) failed to attenuate the influences of stimulus configuration on location-change detection performance. This failure of the posttarget cue for attenuating configural influences on performance may reflect two hypothetical processing limitations: First, when spatial memory load is relatively heavy (e.g., when all three target locations must be encoded into WM because the informative cue is provided only after the target array), attention-control processes may be occupied with rehearsal of the target locations, thereby resulting in reduced attentional resources available for processing the information provided by the posttarget cue. Second, voluntary selective attention may be limited in the degree to which it can suppress well-encoded configural representations in WM. It may be the case that the same information (e.g., irrelevant object locations and overall configuration) can be filtered readily when attention is deployed prior to encoding (as evidenced by the results of Exp. 3), but is difficult to suppress once consolidated into WM. Configurations might be especially subject to these dissociable effects of voluntary attention, because relative locations within the consolidated configuration may be mutually facilitatory, making it difficult to suppress only a subset of locations. Either or both of these possible limitations in the voluntary allocation of attention may underlie the present results. Together, our findings indicate that whereas voluntary selective attention can isolate or prevent the encoding of irrelevant stimulus locations and configural properties, people reliably fail to isolate or suppress configural representations that have been consolidated in WM.

Previous studies (e.g., Delvenne, Cleeremans, \& Laloyaux, 2010; Griffin \& Nobre, 2003; Landman, Spekreijse, \& Lamme, 2003; Lepsien, Griffin, Devlin, \& Nobre, 2005; Matsukura, Luck, \& Vecera, 2007; Schmidt, Vogel, Woodman, \& Luck, 2002) indicated that detection of changes in visual features of objects (e.g., color, orientation, shape) can be facilitated by cues presented before or after the presentation of target arrays. It has been suggested that orienting attention to the cued objects aids in the transfer of their representations into visual WM (e.g., Schmidt et al., 2002) and protects them from degradation or interference during maintenance (Matsukura et al., 2007). However, these studies did not investigate the effects of cueing on the representation of configural information in spatial WM. In general, these previous cueing studies used only few and relatively fixed stimulus locations; there were no controlled manipulations of changes in stimulus configuration from target to probe phases, and in some cases (e.g., Delvenne et al., 2010), measures were even taken to minimize the possibility that relational coding and spatial memory could facilitate the detection of changes in visual features. As a result, it is unknown whether the orienting of selective attention either before or after target presentation can modulate the influences of configuration on detection of changes in spatial location. The present study, in contrast, provides evidence that, whereas allocating spatial attention prior to presentation of a stimulus array can prevent or isolate the encoding of configural information in spatial WM, consolidated configural representations are relatively resistant to attentional modulation. The latter finding stands in stark contrast to the facilitatory effects of postcues found in previous studies of WM for visual features. Together, the results of these studies may reflect greater difficulty in parsing locations bound together within configural representations, as compared to parsing features in other dimensions (e.g., selecting the red object from a set of colored objects held in WM). Future research can be done to investigate the underlying nature of differential postcueing effects for stimulus configurations as compared to other visual features held in WM. 
It may be the case that the representation of configural information provides a natural means for holding locations in mind. Observers may recruit configural or object-based processing mechanisms in order to bolster memory for locations; maintenance of interitem relationships may allow one to reconstruct (e.g., triangulate) individual locations. As a result, even when only a single location is task-relevant, the evaluation of memory for this location may normally be influenced by the nature of the surrounding items, much as the identification of a letter benefits from its being embedded within a word (Reicher, 1969). However, unlike letter identity, individual spatial locations are defined in relation to other items or points of reference. Thus, the profound importance of configural information for defining individual locations, and its potential for bolstering memory for individual locations, may explain why people are in general so robustly influenced by stimulus configuration when evaluating spatial information in WM.

Yet, our findings suggest that perceptual segregation and voluntary selective attention can either suppress the encoding of distractor locations or configural properties into WM, or else isolate the representation of individual target locations from the representation of configural properties. Regarding the latter possibility, observers might maintain parallel configuration-based and location-based (configuration-independent) representations in memory, with the relative weighting of these representations determined by stimulus features (e.g., perceptual segregation afforded by color) and attentional control settings (Folk, Remington, \& Johnston, 1992; Greenberg \& Gmeindl, 2008). Normally, configural representations may be weighted heavily (for the reasons described above), but in some cases attention-control processes might bias these weights (Desimone \& Duncan, 1995) toward the selective processing and maintenance of individual locations at the expense of configural representation. However, the degree to which voluntary attention can bias processing resources toward the representation of individual locations once configural information has been encoded into WM appears to be limited.

\footnotetext{
Author Note L.G. is now at the Department of Psychological and Brain Sciences, Johns Hopkins University; J.K.N. is now at the Derner Institute for Advanced Psychological Studies, Adelphi University. This research was supported by a National Science Foundation Graduate Research Fellowship and a fellowship from the Horace H. Rackham School of Graduate Studies to L.G., a Ruth L. Kirschstein Predoctoral National Research Service Award to J.K.N., and a grant from the National Institutes of Health (R01AG18286) to P.A.R.-L. We thank Jennifer Hsu, Megan Walsh, and Laura Zahodne for assistance with data collection. Supplemental materials may be downloaded along with this article from www.springerlink.com.
}

\section{References}

Awh, E., Anllo-Vento, L., \& Hillyard, S. A. (2000). The role of spatial selective attention in working memory for locations: Evidence from event-related potentials. Journal of Cognitive Neuroscience, 12, 840-847. doi:10.1162/089892900562444

Awh, E., Jonides, J., \& Reuter-Lorenz, P. A. (1998). Rehearsal in spatial working memory. Journal of Experimental Psychology. Human Perception and Performance, 24, 780-790. doi:10.1037/ 0096-1523.24.3.780

Awh, E., Jonides, J., Smith, E. E., Buxton, R. B., Frank, L. R., Love, T., et al. (1999). Rehearsal in spatial working memory: Evidence from neuroimaging. Psychological Science, 10, 433-437. doi:10.1111/1467-9280.00182

Boduroglu, A., Gmeindl, L., \& Reuter-Lorenz, P. A. (2011). Flexible configural processing is preserved in old age: Evidence from spatial working memory. Manuscript submitted for publication. Turkey: Bogazici University.

Bor, D., Duncan, J., \& Owen, A. M. (2001). The role of spatial configuration in tests of working memory explored with functional neuroimaging. Scandinavian Journal of Psychology, 42, 217-224. doi:10.1111/1467-9450.00232

Bor, D., Duncan, J., Wiseman, R. J., \& Owen, A. M. (2003). Encoding strategies dissociate prefrontal activity from working memory demand. Neuron, 37, 361-367. doi:10.1016/S0896-6273(02) 01171-6

Delvenne, J.-F., \& Bruyer, R. (2006). A configural effect in visual short-term memory for features from different parts of an object. Quarterly Journal of Experimental Psychology, 59, 1567-1580. doi:10.1080/17470210500256763

Delvenne, J.-F., Cleeremans, A., \& Laloyaux, C. (2010). Feature bindings are maintained in visual short-term memory without sustained focused attention. Experimental Psychology, 57, 108116. doi:10.1027/1618-3169/a000014

Desimone, R., \& Duncan, J. (1995). Neural mechanisms of selective visual attention. Annual Review of Neuroscience, 18, 193-222. doi:10.1146/annurev.ne.18.030195.001205

Duncan, J., \& Humphreys, G. W. (1989). Visual search and stimulus similarity. Psychological Review, 96, 433-458. doi:10.1037/0033295X.96.3.433

Egeth, H. E., \& Yantis, S. (1997). Visual attention: Control, representation, and time course. Annual Review of Psychology, 48, 269-297. doi:10.1146/annurev.psych.48.1.269

Folk, C. L., Remington, R. W., \& Johnston, J. C. (1992). Involuntary covert orienting is contingent on attentional control settings. Journal of Experimental Psychology. Human Perception and Performance, 18, 1030-1044. doi:10.1037/ 0096-1523.18.4.1030

Gmeindl, L., Walsh, M., \& Courtney, S. M. (2011). Binding serial order to representations in working memory: A spatial/verbal dissociation. Memory \& Cognition, 39, 37-46. doi:10.3758/ s13421-010-0012-9

Greenberg, A. S., \& Gmeindl, L. (2008). Strategic control of attention to objects and locations. Journal of Neuroscience, 28, 564-565. doi:10.1523/JNEUROSCI.4386-07.2008

Griffin, I. C., \& Nobre, A. C. (2003). Orienting attention to locations in internal representations. Journal of Cognitive Neuroscience, 15, 1176-1194. doi:10.1162/089892903322598139

Jha, A. P. (2002). Tracking the time-course of attentional involvement in spatial working memory: An event-related potential investigation. Cognitive Brain Research, 15, 61-69. doi:10.1016/S09266410(02)00216-1

Jiang, Y., Chun, M. M., \& Olson, I. R. (2004). Perceptual grouping in change detection. Perception \& Psychophysics, 66, 446-453. doi:10.3758/BF03194892 
Jiang, Y., Olson, I. R., \& Chun, M. M. (2000). Organization of visual short-term memory. Journal of Experimental Psychology. Learning, Memory, and Cognition, 26, 683-702. doi:10.1037/02787393.26.3.683

Landman, R., Spekreijse, H., \& Lamme, V. A. F. (2003). Large capacity storage of integrated objects before change blindness. Vision Research, 43, 149-164.

Lepsien, J., Griffin, I. C., Devlin, J. T., \& Nobre, A. C. (2005). Directing spatial attention in mental representations: Interactions between attentional orienting and working-memory load. NeuroImage, 26, 733-743.

Lepsien, J., \& Nobre, A. C. (2006). Cognitive control of attention in the human brain: Insights from orienting attention to mental representations. Brain Research, 1105, 20-31.

Matsukura, M., Luck, S. J., \& Vecera, S. P. (2007). Attention effects during visual short-term memory maintenance: Protection or prioritization? Perception \& Psychophysics, 69, 1422-1434. doi:10.3758/BF03192957

Munneke, J., Heslenfeld, D. J., \& Theeuwes, J. (2010). Spatial working memory effects in early visual cortex. Brain and Cognition, 72, 368-377. doi:10.1016/j.bandc.2009.11.001

Postle, B. R., Awh, E., Jonides, J., Smith, E. E., \& D'Esposito, M. (2004). The where and how of attention-based rehearsal in spatial working memory. Cognitive Brain Research, 20, 194 205.
Reicher, G. M. (1969). Perceptual recognition as a function of meaningfulness of stimulus material. Journal of Experimental Psychology, 81, 275-280.

Schmidt, B. K., Vogel, E. K., Woodman, G. F., \& Luck, S. J. (2002). Voluntary and automatic attentional control of visual working memory. Perception \& Psychophysics, 64, 754-763. doi:10.3758/ BF03194742

Smyth, M. M. (1996). Interference with rehearsal in spatial working memory in the absence of eye movements. Quarterly Journal of Experimental Psychology, 49A, 940-949.

Smyth, M. M., \& Scholey, K. A. (1994). Interference in immediate spatial memory. Memory \& Cognition, 22, 1-13.

Tanner, W. P., Jr., \& Swets, J. A. (1954). A decision-making theory of visual detection. Psychological Review, 61, 401-409. doi: $10.1037 / \mathrm{h} 0058700$

Van der Stigchel, S., Merten, H., Meeter, M., \& Theeuwes, J. (2007). The effects of a task-irrelevant visual event on spatial working memory. Psychonomic Bulletin \& Review, 14, 1066-1071. doi:10.3758/BF03193092

Vogel, E. K., Woodman, G. F., \& Luck, S. J. (2006). The time course of consolidation in visual working memory. Journal of Experimental Psychology. Human Perception and Performance, 32, 1436-1451.

Woodman, G. F., Vecera, S. P., \& Luck, S. J. (2003). Perceptual organization influences visual working memory. Psychonomic Bulletin \& Review, 10, 80-87. doi:10.3758/BF03196470 